\title{
Tensión Muscular Percibida en Operarias de Máquinas de Moldeo por Inyección de Plástico
}

\section{PERCEIVED MUSCLE TENSION IN FEMALE WORKERS OF PLASTIC INJECTION MOLDING MACHINES}

\author{
Douglas Alayón'1, Yohama Caraballo-Arias², Yaineth Rivera Trivison ${ }^{3}$ \\ 1. OHSE Coordinator Aggreko de Venezuela. \\ 2. Docente e investigadora, Facultad de Medicina, Universidad Central de Venezuela. \\ 3. Centro Médico Docente de La Trinidad.
}

\begin{abstract}
RESUMEN
Objetivo: Identificar la incidencia de Tensión Muscular Percibida (TMP) según edad, antigüedad y tipo de jornada en operarias de máquinas de moldeo por inyección de plástico. Métodos: se aplicó el cuestionario Nórdico Estandarizado a 86 de 128 trabajadoras con mínimo 1 año de antigüedad. Resultados: Según el estudio transversal, el 84,9\% de trabajadoras presentó TMP, con mayor incidencia en aquellas de 40 años de edad o más $(90,2 \%)$, y mayor aun en el grupo de 6 a 10 años de antigüedad en el puesto (93,2\%). Respecto al tipo de jornada laboral, la mayor incidencia de TMP fue en trabajadoras del horario nocturno $(95,2 \%)$. El segmento corporal más afectado fue el cuello (63\%), seguido por hombros y manos/muñecas $(60,3 \%$, respectivamente), y columna lumbar (50,7\%). Del total de operarias con TMP, el 64,4\% refirió impedimento para el desempeño de su trabajo en el último año, mientras que el 80,8\% indicó manifestaciones corporales durante la última semana. Conclusiones: No hubo significancia estadística de TMP con respecto a edad (X2 = 1,798; $\mathrm{p}$ $>0,05)$ o jornadas de trabajo $(\mathrm{X} 2=3,636 ; \mathrm{p}>0,05)$, pero sí en relación con antigüedad (X2 =13,881; $\mathrm{p}<0,05)$. Las posturas estáticas y movimientos repetitivos pueden ser algunos factores de riesgo ocupacionales causales de TMP.
\end{abstract}

(Alayón D, Caraballo-Arias Y, Rivera Y, 2015. Tensión Muscular Percibida en Operarias de Máquinas de Moldeo por Inyección de Plástico. Cienc Trab. May-Ago; 17 [53]: 137-143).

Palabras clave: RIESGOS LABORALES, ENFERMEDADES PROFESIONALES, TRASTORNOS DE TRAUMAS ACUMULADOS, SISTEMA MÚSCULO-ESQUELÉTICO.

\section{ABSTRACT}

Objective: To identify relationships between Perceived Muscular Tension (PMT), age, time-on-job, and labor shifts for female operators of plastic molding injection machines. Methods: a standardized Nordic questionnaire was administered to 86 of the 128 workers with at least 1 year on job. Results: Cross-sectional study, of an average population of 39.9 years of age, yielded $84.9 \%$ of workers affected with PMT, with a higher incidence amongst individuals 40 years or older (90.2\%). The highest incidence of affected individuals was noticed in the group with 6 to 10 years on the job (93.2\%). With regards to work shift, the highest prevalence of TMP was amongst night shift workers (95.2\%). Regarding the affected body part ; neck (63\%), followed by: (i) shoulders and (ii) hands / wrists (both with $60.3 \%$ ), and lower back (50.7\%). From those that reported PMT, $64.4 \%$ reported some impairment in the performance of their work duties in the past year, while $80.8 \%$ indicated having symptoms during the immediately preceding week. Conclusions: as it relates to PMT, there is no statistical significance for the variables age (X2 = $1.798, \mathrm{p}>0.05)$ or work shift $(\mathrm{X} 2=3.636, \mathrm{p}>0.05)$. However, timeon-job (X2 = 13.881; $\mathrm{p}<0.05)$ has statistical relevance.

Keywords: OCCUPATIONAL RISKS, OCCUPATIONAL DISEASES, CUMULATIVE TRAUMA DISORDERS, MUSCULOSKELETAL SYSTEM.

\section{INTRODUCCIÓN}

Los trastornos músculo-esqueléticos (TME) comprenden un conjunto de condiciones que incluyen afectación de carácter inflamatorio y/o degenerativo de los elementos del aparato loco-

Correspodencia / Correspondence:

Yohama Caraballo-Arias

Universidad Central de Venezuela, Ciudad Universitaria, Los Chaguaramos

Caracas, Venezuela.

Tel.: (0058)-426-5187210.

e-mail: yohama.caraballo@ucv.ve

Recibido: 22 de Febrero 2015 / Aceptado: 28 de Mayo de 2015 motor, pudiendo diferenciarse desde sintomas reiterados leves (por ejemplo: dolor, entumecimiento, parestesia y molestia en una o varias regiones corporales), hasta condiciones incapacitantes crónicas severas, que implican dificultad para la ejecución habitual de tareas laborales o de otra índole de modo temporal o permanente. ${ }^{1-5}$

En cuanto a los elementos fisiopatológicos relacionados con la aparición de los TME, la evidencia científica señala que son de naturaleza multifactorial. En este sentido, se puede considerar cuatro grandes grupos de riesgo, que son: factores individuales (capacidad funcional del trabajador, hábitos y antecedentes), factores de carga física (fuerza, posturas y movimientos), factores psicosociales (organización del trabajo, jornadas, horarios, pausas, ritmo y carga de trabajo, entre otros) y factores ambientales (temperatura, vibración, entre otros). ${ }^{3,4}$ 
En muchos países los TME constituyen una proporción importante de todas las enfermedades relacionadas con el trabajo; por ejemplo, en Venezuela representa el 90\% de las enfermedades (Instituto Nacional de Prevención, Salud y Seguridad Laborales), ${ }^{6,7}$ mientras que en los Estados Unidos, los países nórdicos y Japón, representa un tercio o más de las enfermedades ocupacionales registradas. ${ }^{8}$ Algunos de los TME más comunes relacionados con el trabajo son el lumbago, la cervicalgia, la tendinitis del brazo y el hombro, y el síndrome del túnel carpiano. ${ }^{9}$

El surgimiento del trabajo industrializado asociado a una búsqueda desenfrenada por la productividad ha tenido efectos negativos sobre el bienestar de los trabajadores. ${ }^{10.11}$ Una de las industrias con mayor relevancia en el mundo es la del plástico, ya que este material está presente en casi todos los productos industriales y de consumo, haciendo la vida moderna prácticamente inconcebible sin él. La producción durante los últimos diez años ha sido equivalente a la de todo el siglo XX. Se calcula que la producción mundial de plásticos podría triplicarse en $2050 .{ }^{12}$

Dentro del sector del plástico se pueden distinguir 2 tipos de industrias: productoras de material plástico (que producen polímeros) y las empresas dedicadas a la fabricación o manufactura de piezas plásticas. ${ }^{13}$ Estas últimas siguen diversos procesos de producción, entre los cuales se encuentran: el almacenamiento de materias primas y productos necesarios para la fabricación, moldeo de plástico, la matricería (donde se revisan y reparan los moldes matrices para la producción, así como los servicios generales y auxiliares de producción). ${ }^{13}$ Cada uno de los procesos llevados a cabo en este tipo de industria tiene riesgos propios e influencia en la etiología de enfermedades ocupacionales de origen músculo-esquelético según la naturaleza de las tareas que se realizan. El proceso neurálgico de una industria de manufactura de piezas plásticas es el moldeo del plástico, siendo una de las técnicas más utilizadas el de inyección, que consiste en embeber un polímero fundido en un molde cerrado y frío, donde se solidifica por un breve tiempo para dar el producto. La pieza moldeada se recupera al abrir el molde para expulsarla. ${ }^{14}$ Un esquema de una máquina de moldeo por inyección puede apreciarse en la Figura $\mathrm{N}^{\circ} 1$.
A veces las piezas expulsadas por la máquina de moldeo por inyección de plástico deben ser refinadas o acabadas posteriormente para eliminar rebabas (residuo resultante de la introducción del plástico fundido en la superficie de separación entre las partes del molde) o cortar las coladas (residuo procedente del enfriamiento del material contenido en el conducto de inyección al molde. ${ }^{15}$ Estas actividades se efectúan de modo manual y repetitivo, frecuentemente bajo postura estáticas prolongadas, lo cual pudiera contribuir con la aparición TME en el sector de la industria del plástico. ${ }^{16}$

Por otra parte, el género constituye un factor que también tiene influencia en la aparición de TME, ya que en la mayoría de los estudios estos trastornos resultan más frecuentes en las mujeres que en los hombres. ${ }^{17}$ Es importante reconocer que aquellos trabajos considerados livianos, y que normalmente realiza la mujer, pueden exigir un grado de resistencia muscular, en especial cuando se realizan en posturas estáticas del cuerpo y con movimientos repetidos en manos y brazos durante la mayor parte de la jornada laboral ${ }^{18}$, ratificando de esta manera la naturaleza multifactorial de los TME.

Asimismo, existen varias investigaciones sobre la correlación entre la presencia de TME y otros factores individuales como la edad y antigüedad laboral. La incidencia de síntomas de TME en ubicaciones anatómicas específicas aparecen con mayor frecuencia en trabajadores adultos mayores, incluso en aquellos casos de trabajos considerados poco exigentes, lo cual pareciera indicar efectos acumulativos del riesgo a largo plazo. ${ }^{19}$ En este sentido, pareciera existir una importante correlación entre la edad de los trabajadores y los años de trabajo, resultando complicado determinar si el factor de riesgo es únicamente la edad, la antigüedad laboral, o ambos..$^{20,21}$ No obstante, no todas las investigaciones han obtenido resultados positivos de la asociación de la edad con los TME. ${ }^{22}$

Con respecto a la influencia de los turnos de trabajo sobre la incidencia de los TME, existen pocas investigaciones. ${ }^{23}$ De hecho, la mayoría de los estudios realizados sobre las consecuencias de los turnos de trabajo sobre la salud se orientan más hacia trastornos nerviosos, fatiga, trastornos del sueño, e incluso trastornos psicológicos, gastrointestinales, cardiovasculares y metabólicos.

Figura 1.

Esquema de máquina de moldeo de plástico por inyección.

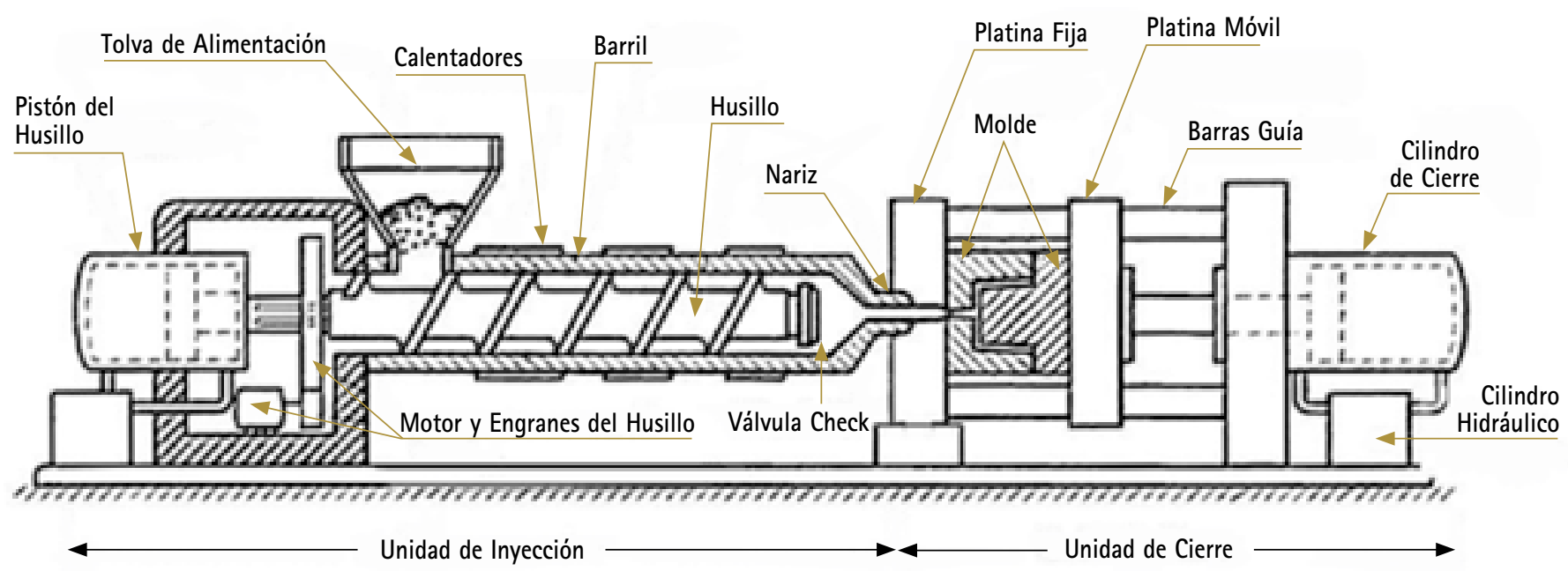

Fuente: http://tecnologiadelosplasticos.blogspot.com/2011/06/inyeccion-de-materiales-plasticos-i.html 
El propósito principal de este estudio fue determinar la incidencia de TMP y los factores asociados a la misma entre operarias de máquinas de moldeo por inyección de plástico.

\section{MATERIAL Y MÉTODOS}

Estudio descriptivo de corte transversal realizado en una población de trabajadoras bajo el cargo de operarias de máquinas para el moldeo por inyección, pertenecientes a una empresa manufacturera de productos plásticos de la ciudad de Caracas (Venezuela) en julio de 2013. La población del estudio estuvo constituida por 128 mujeres, pero solo se aplicó a 86 de las mismas $(n=86)$, conforme a los criterios de inclusión y exclusión.

Criterios de inclusión: se incluyeron las trabajadoras de moldeo por inyección con un tiempo mínimo de vinculación a la empresa $\mathrm{y}$ al cargo de 12 meses.

Criterios de exclusión: se excluyeron todas las trabajadoras que no consintieron voluntariamente su participación en el estudio, así como aquellas no presentes al momento del mismo por causa de cualquier indole.

No se consideró extensible el estudio a trabajadores del sexo masculino, dada la diferenciación de responsabilidades y funciones evidentes con respecto al sexo femenino.

Para la recolección de los datos se aplicó a las trabajadoras una versión traducida al español del Cuestionario Nórdico Estandarizado $^{24,25}$, el cual permite evaluar la presencia de sintomas músculoesqueléticos en nueve regiones del cuerpo (cuello, hombros, codos, muñecas/manos, zona dorsal, zona lumbar, caderas, rodillas y tobillos/pies) durante los 12 meses previos a la aplicación del cuestionario. La respuesta afirmativa generó preguntas adicionales con respecto al impedimento para realizar sus funciones laborales de modo normal por causa del síntoma, y si este había estado presente en algún momento en los últimos 7 días. El cuestionario incluyó un diagrama corporal para facilitar la identificación de las regiones corporales.

El Cuestionario Nórdico determina algunas condiciones individuales y laborales de la población en estudio en relación con la tensión muscular percibida y aspectos relacionados con la edad, antigüedad en el cargo y tipo de jornada laboral (diurna, nocturna o mixta).

El análisis de la información se centró en el cálculo de proporciones descriptivas, para lo cual se utilizó el programa Microsoft Excel 2010 para la recolección de la información, y el análisis se llevó a cabo mediante el software SPSS Statistics 20. Los resultados se expresaron en valores absolutos y relativos porcentuales, se calculó la media y desviación estándar a las variables edad, antigüedad y tipo de jornada laboral. Para relacionar la presencia de síntomas con las variables individuales y laborales se empleó la prueba Chi cuadrado de Pearson, considerándose estadísticamente significativos los resultados con una $\mathrm{p}<0,05$.

\section{RESULTADOS}

La edad promedio de las trabajadoras fue de 39,5 \pm 9,2 años (la trabajadora de menor edad fue de 20 y la mayor de 64 años) y un promedio de antigüedad de 8,83 \pm 6,56 años (el tiempo laborado en la empresa estuvo entre 1 y 37 años), distribuidas en la jornada diurna, 33,72\%; nocturna, 24,42\%; y mixta, 41,86\%.

El $84,9 \%(n=73)$ de las trabajadoras presentó TMP en los últimos 12 meses, cuya edad promedio fue de 39,9 \pm 9 años (rango de 20 a 64 años) y una antigüedad media de 8,49 \pm 5,74 años (rango de 2 a 36 años), distribuidas en jornada laboral diurna $(30,14 \%)$, nocturna $(27,40 \%)$ y mixta $(42,46 \%)$.

La mayor incidencia de TMP se observa en trabajadoras de 40 a 49 años $(90,3 \%)$ y en el grupo de 50 años o más $(90,0 \%)$ (Tabla $\left.\mathrm{N}^{\circ} 1\right)$. Al combinar ambos grupos etarios se verifica una incidencia de 90,2\% para trabajadoras con TMP de 40 o más años de edad. Al aplicar la prueba Chi cuadrado de Pearson no se obtuvo diferencia significativa entre la edad y la presencia de TMP $\left(X^{2}=1,798 p=0,615\right)$.

Al evaluar la existencia de TMP según la antigüedad en el puesto de trabajo, la mayor incidencia se observa inicialmente en los

Tabla 1.

Incidencia de tensión muscular percibida en operarias de máquinas estratificada por grupos etarios. Caracas, 2013.

\begin{tabular}{lcccccc|} 
Grupo Etario & N & $\%$ & \multicolumn{5}{c}{ Presencia de signos asociados a TMP } \\
& & & Sí & NO \\
29 años o menos & 9 & 10,5 & 7 & 77,8 & 2 & 22,2 \\
30-39 años & 36 & 41,9 & 29 & 80,6 & 7 & 19,4 \\
40-49 años & 31 & 36 & 28 & 90,3 & 3 & 9,7 \\
50 años o más & 10 & 11,6 & 9 & 90 & 1 & 10 \\
\hline
\end{tabular}

$X^{2}=1,798 p>0,05$

Fuente: Datos de la investigación. Julio, 2013.

grupos de trabajadoras de 16 a 20 años y 26 a 30 años de antigüedad. Sin embargo, al analizar los grupos mayores de 16 años de antigüedad, se observa que la frecuencia de los sujetos con TMP es 7 veces menor que la verificada para grupos de menor antigüedad (ver Gráfico $\mathrm{N}^{\circ} 1$ ), lo cual pudiera conllevar a errores de interpretación global de los resultados.

En el gráfico se verifica la mayor incidencia de TMP en trabajadoras con menos de 10 años de antigüedad (88,2\%), más específicamente en el grupo entre 6 y 10 años de desempeño en el cargo (93,2\%).

$\mathrm{Al}$ aplicar la prueba de independencia de Chi cuadrado se determinó la relación significativa entre la variable antigüedad y la presencia de TMP $\left(X^{2}=13,381 \mathrm{p}=0,031\right)$ concluyéndose que las variables antigüedad y presencia de indicios de TMP están relacionadas.

En relación de TMP según el tipo de jornada de trabajo, la mayor incidencia de sintomas se observa en trabajadoras que laboran en jornada nocturna $(95,2 \%)$, seguidas por la jornada mixta $(86,1 \%) \mathrm{y}$, finalmente, la jornada diurna (75,9\%). Al aplicar la prueba de Chi

\section{Gráfico 1.}

Distribución por antigüedad de operarias de máquinas totales y con TMP en los últimos 12 meses. Caracas, 2013.

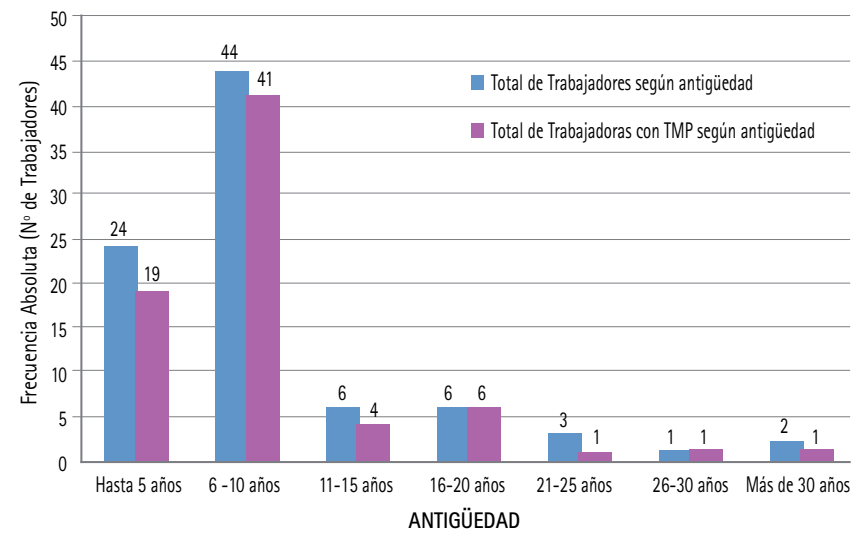

Fuente: Datos de la investigación. Julio, 2013. 
Tabla 2

Incidencia de tensión muscular percibida en operarias de máquinas según tipo de jornada laboral. Caracas, 2013.

\begin{tabular}{|c|c|c|c|c|c|c|}
\hline \multirow{3}{*}{$\begin{array}{l}\text { Tipo de } \\
\text { Jornada Laboral }\end{array}$} & \multirow[t]{3}{*}{$\mathrm{N}$} & \multirow[t]{3}{*}{$\%$} & \multicolumn{4}{|c|}{ Presencia de signos asociados a TMP } \\
\hline & & & \multirow{2}{*}{$\begin{array}{l}\text { Sí } \\
\mathrm{N}\end{array}$} & \multicolumn{3}{|c|}{ NO } \\
\hline & & & & $\%$ & $\mathrm{~N}$ & $\%$ \\
\hline Diurna & 29 & 33,7 & 22 & 75,9 & 7 & 24,1 \\
\hline Mixta & 36 & 41,9 & 31 & 86,1 & 5 & 13,9 \\
\hline Nocturna & 21 & 24,4 & 20 & 95,2 & 1 & 4,8 \\
\hline
\end{tabular}

$X^{2}=3,636 p>0,05$

Fuente: Datos de la investigación. Julio, 2013

Cuadrado de Pearson no se obtuvo diferencia significativa entre el tipo de jornada laboral y la presencia de TMP $\left(X^{2}=3,636 \mathrm{p}=0,162\right)$. En los últimos 12 meses, el 82,2\% $(n=60)$ de las trabajadoras manifestó tener TMP en múltiples regiones, mientras que el 17,8\% ( $\mathrm{n}=13$ ) en una única región anatómica. Por otra parte, el 64,4\% ( $n=47)$ refirió impedimento para el desempeño de su trabajo en el último año, mientras que el 80,8\% $(n=59)$ indicó algún tipo de TMP durante la última semana.

En el Gráfico $\mathrm{N}^{\circ} 2$ se aprecia la relación de la frecuencia relativa en las regiones del cuerpo más comúnmente afectadas en el último año. El cuello representa la incidencia más alta $(63,0 \%)$, seguido por hombros y manos/muñecas (60,3\%, respectivamente), y en tercer lugar la columna lumbar (50,7\%). Las encuestadas informaron TMP con menor frecuencia en caderas/piernas (39,7\%), columna dorsal $(37,0 \%)$, rodillas $(34,2 \%)$, codos $(32,9 \%)$ y los tobillos/pies $(31,5 \%)$.

En el Gráfico $\mathrm{N}^{\circ} 3$ se aprecia la relación de la frecuencia relativa en las regiones del cuerpo más afectadas en los últimos 7 días. El cuello representa la incidencia más alta (59,3\%), seguido por manos/muñecas (49,2\%), y en tercer lugar los hombros (47,5\%). Las encuestadas manifestaron TMP con menor frecuencia en columna lumbar (40,7\%), caderas/piernas (35,6\%), tobillos/pies $(32,2 \%)$, rodillas $(23,7 \%)$, y finalmente, columna dorsal y codos (22\%, respectivamente).

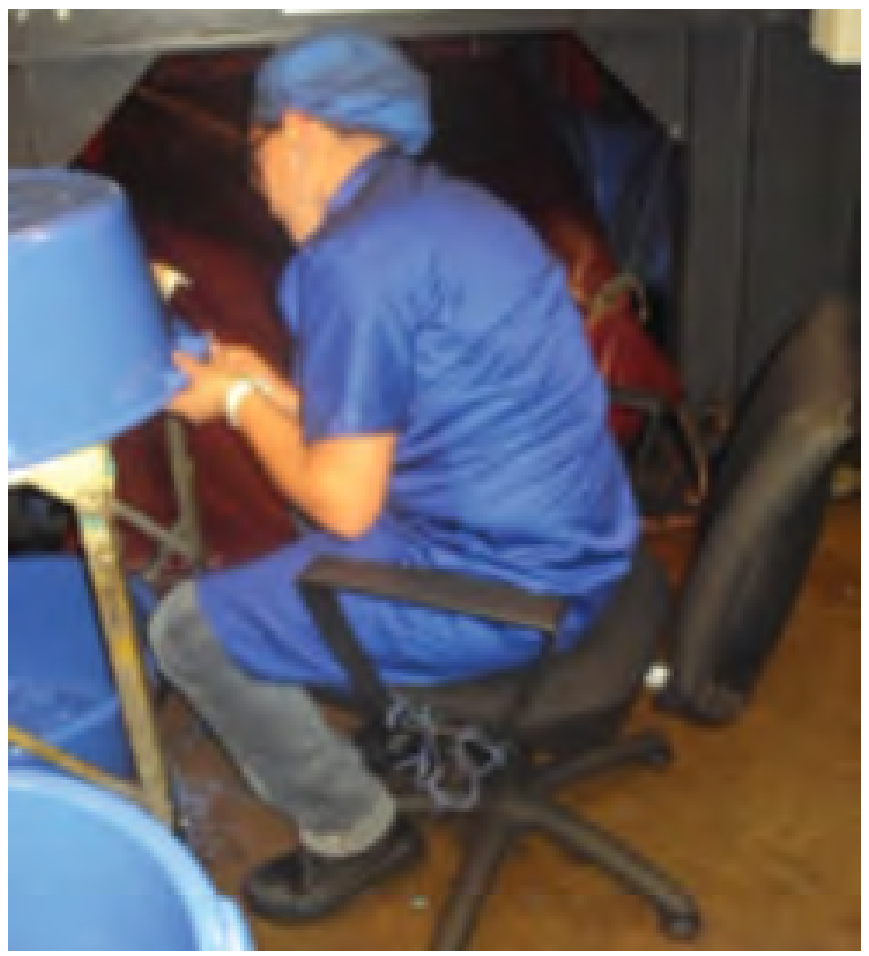

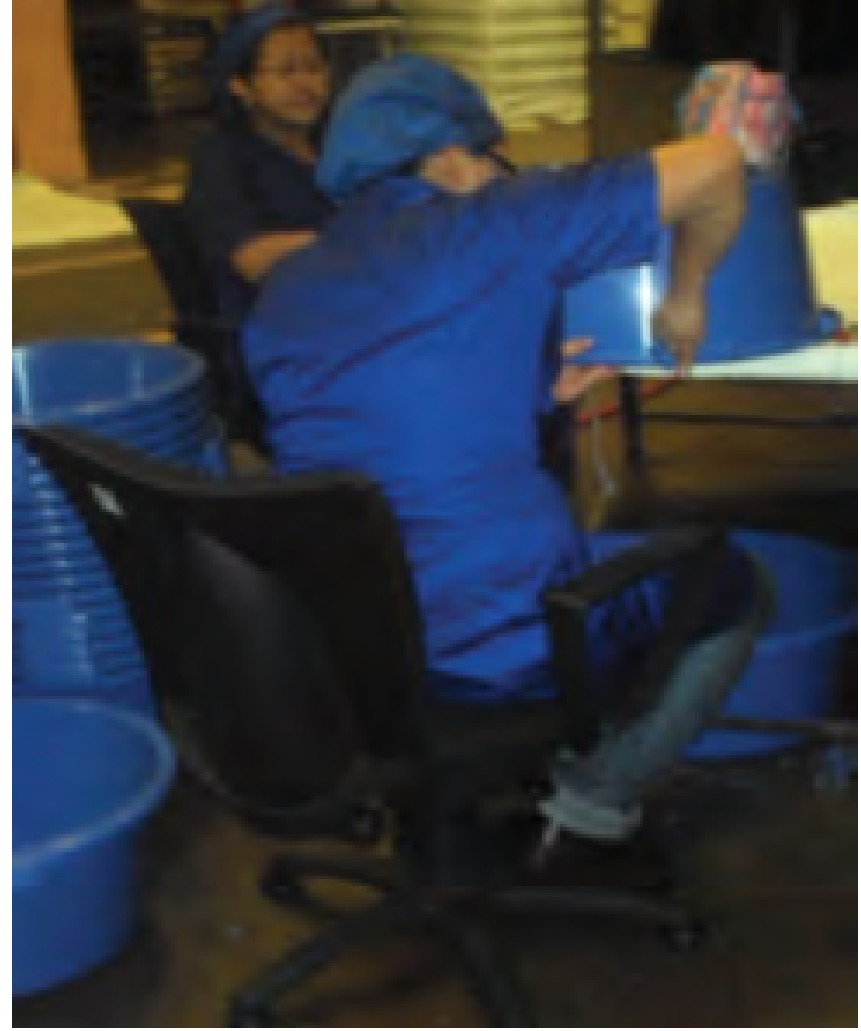

Gráfico 2.

Distribución de la frecuencia relativa de TMP en los últimos 12 meses según ubicación anatómica. Caracas, 2013.

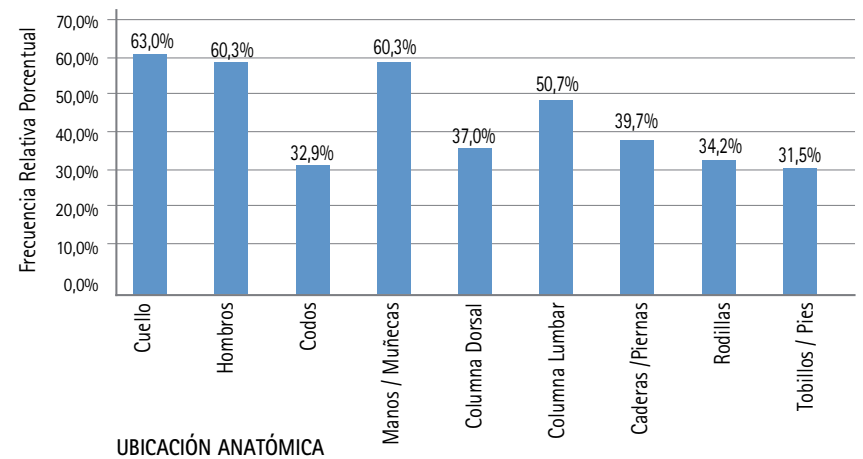

Gráfico 3.

Incidencia de signos de TMP en los últimos 7 dias según ubicación. Caracas, 2013.

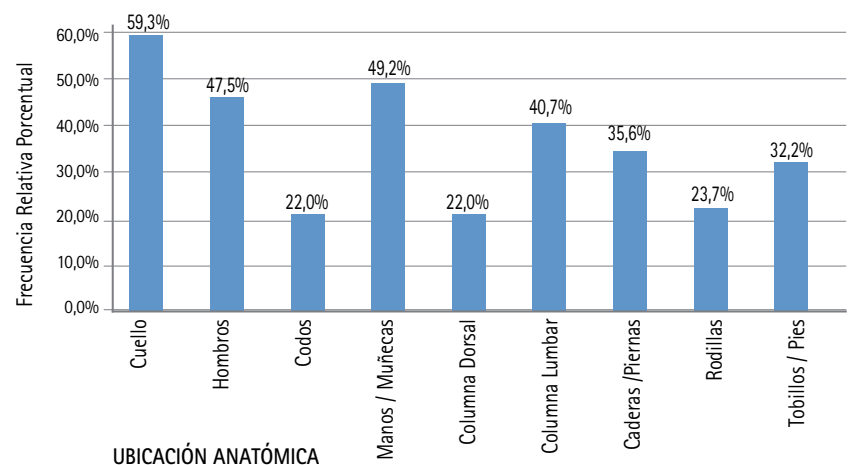

Fuente: Datos de la investigación. Julio, 2013. 


\section{Gráfico 4.}

Incidencia de impedimento laboral en los últimos 12 meses según ubicación anatómica en las operarias de máquinas. Caracas, 2013.

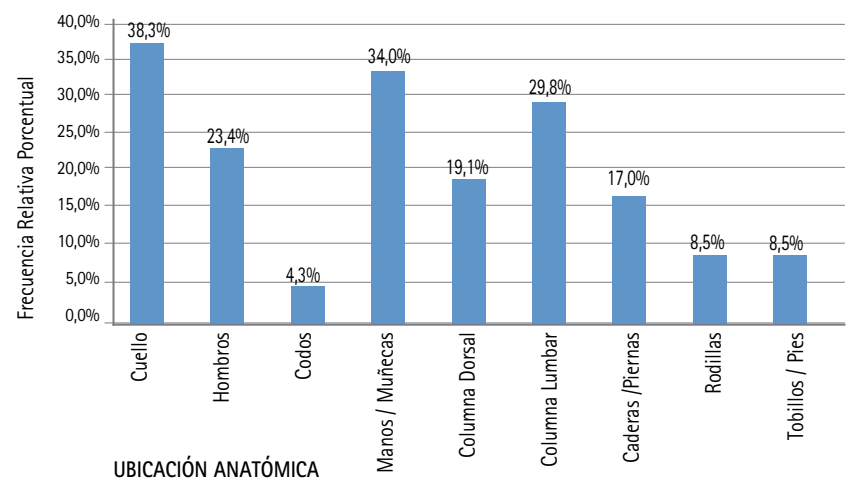

Fuente: Datos de la investigación. Julio, 2013.

En el Gráfico $\mathrm{N}^{\circ} 4$ se representa el impedimento para trabajar en los últimos 12 meses, donde el cuello tiene la incidencia más alta (38,3\%), seguida del impedimento en manos/muñecas (34\%) y columna lumbar (29,8\%). Las encuestadas informaron de los TMP de menor incidencia fueron en hombros $(23,4 \%)$, columna dorsal $(19,1 \%)$, caderas/piernas (17\%), rodillas y tobillos/pies $(8,5 \%), y$, finalmente, codos $(4,3 \%)$.

\section{DISCUSIÓN Y CONCLUSIONES}

En la industria de manufactura de piezas plásticas, las operarias de máquinas de moldeo por inyección están encargadas del corte de coladas frías, la eliminación de rebabas y, en ocasiones, del ensamblaje y etiquetado de los productos elaborados, lo que implica la exposición a una serie de factores de carga física que pueden contribuir a la presentación de trastornos músculo-esqueléticos, tal como se evidenció en la alta incidencia $(84,9 \%)$ de tensión muscular percibida (TMP) de la población estudiada. Este hallazgo es comparable con un estudio similar realizado entre 577 trabajadores de 14 compañías del sector de la industria plástica en Brasil, el cual registró una incidencia general de síntomas músculoesqueléticos de 64\% (17), mientras que el mismo estudio aplicado solo a la población femenina arrojó un 67\% de incidencia de TMP. Con respecto a la variable edad, no se encontró relación significativa entre los sintomas músculo-esqueléticos y la edad, al utilizar la prueba Chi cuadrado de Pearson. La mayor incidencia de TMP se ubicó en trabajadoras adultas maduras, destacando el grupo de 40 a 49 años, observándose una cuasi-estabilidad de la misma a partir de los 50 años de edad. Esto podría deberse a que las trabajadoras de mayor edad -no capaces de soportar la carga física propia de sus funciones laborales, debido a molestias músculo-esqueléticas- no forman parte del grupo estudiado, bien sea por terminación de la relación laboral, o bien por cambio a una actividad laboral con menor demanda física. $^{26}$ Sin embargo, no puede descartarse la incidencia de efectos acumulativos del riesgo a largo plazo, dada la incidencia de las acciones repetitivas con ciclos de trabajo cortos y posturas estáticas prolongadas, incluso en aquellos casos de trabajos considerados poco exigentes $^{27}$, con o sin apoyo de otros trabajadores.

Por otra parte, se determinó que existe asociación significativamente estadística entre la antigüedad y la presencia de TMP, al valerse de la prueba Chi cuadrado de Pearson. La principal inci- dencia de TMP se constató en el grupo entre 6 y 10 años de desempeño en el cargo $(93,2 \%)$, con posterior disminución en incidencia y frecuencia para trabajadoras con más de 10 años de antigüedad. Una posible explicación a estos resultados es que las trabajadoras del grupo entre 6 y 10 años de antigüedad, específicamente las de 7 años de antigüedad (que corresponde a la moda y mediana de dicha variable), pudieron estar expuestas a condiciones laborales diferentes en ese período (por ejemplo: tecnología del proceso productivo, implementación de herramientas específicas de trabajo, etc.) con respecto a los trabajadores actuales, mientras que la disminución de la incidencia de TMP antes de ese lapso de tiempo, es decir a las de menor antigüedad, pudiera asociarse a menor exposición a los factores de riesgo y a la adecuación tecnológica de los procesos. Por su parte, el decremento de incidencia de TMP en trabajadoras con antigüedad mayor a 10 años podría deberse al cambio en sus métodos y técnicas de trabajo manual (más eficientes y seguras) para poder sobrellevar las cargas físicas de trabajo. ${ }^{26}$ Asimismo, hay que considerar la capacidad de las trabajadoras con mayor antigüedad (que generalmente coinciden con las de más edad) para desarrollar mecanismos de protección frente a los TME. ${ }^{28}$

No se encontró relación significativa entre el tipo de jornada y TMP al utilizar la prueba Chi cuadrado de Pearson. No obstante, la mayor incidencia de TMP se ubicó en trabajadoras nocturnas. Esto podría asociarse al problema de la resincronización de las funciones fisiológicas después de un cambio en las horas laborales y de sueño, al que hace mención La Dou y sus colaboradores. ${ }^{19,29}$ Puesto que en promedio los trabajadores diurnos descansan 7 horas, mientras que los trabajadores nocturnos duermen poco más de 4 horas, según un estudio realizado por Khalequeen en $1999^{30}$, lo cual les permite disponer de tiempo adicional para desempeñar otro tipo de labores, aun en detrimento de su salud. Ahora bien, si se considera los otros roles de la mujer (madre, ama de casa), sería lógico pensar que le resulta más difícil soportar los efectos del trabajo en turnos nocturnos. La carga física adicional, las pocas

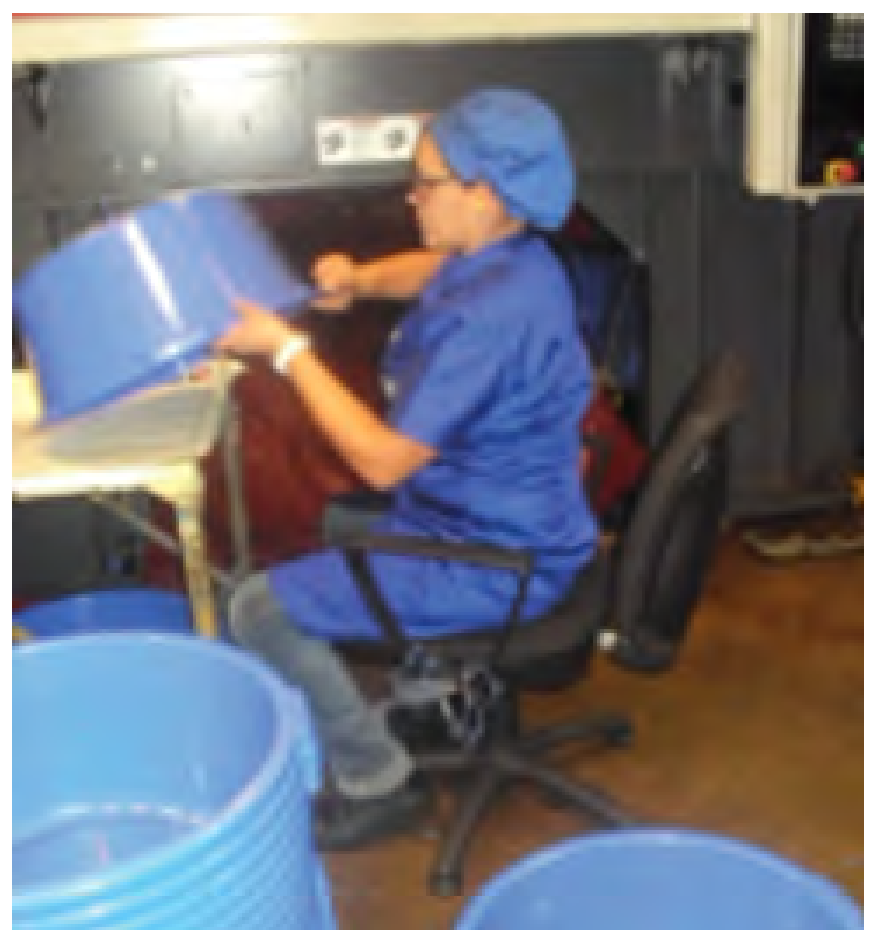


horas de descanso y los efectos colaterales de estresores psicosociales (laborales, extra-laborales y personales), junto al trabajo nocturno, propician la aparición de los TME.

La relación entre los TME y los factores psicosociales es compleja dada la gran variedad de situaciones posibles, sobre todo los vinculados con factores personales. ${ }^{21}$ La asociación entre los factores psicosociales y los TME son, en primer lugar, que las exigencias psicosociales pueden producir un aumento de la tensión muscular y exacerbar el esfuerzo biomecánico de la tarea; en segundo lugar, que las exigencias psicosociales pueden afectar a la conciencia y al informe sobre los síntomas músculo-esqueléticos y/o a la percepción de sus causas; en tercer lugar, que los episodios de dolor provocados por factores físicos pueden causar disfunciones crónicas en el sistema nervioso, tanto fisiológicas como psicológicas, que mantengan procesos crónicos de dolor. ${ }^{21}$ Así pues, los TME pueden ser consecuencia de las exigencias físicas o de las psicosociales o del efecto modificador de la relación entre ambas.

En los últimos 12 meses, tanto como en los 7 días previos a la aplicación del Cuestionario Nórdico, las regiones anatómicas frecuentemente más afectadas en las trabajadoras de este estudio son: cuello, hombros, manos/muñecas y zona lumbar, las cuales también fueron causa de impedimento de actividades laborales durante el último año. Es importante señalar que las posturas estáticas o forzadas, así como los movimientos repetitivos pueden ser algunos riesgos ocupacionales causales de los TME, sin que ello implique desestimación de los grupos de riesgos restantes, sobre todo si se tiene en cuenta la naturaleza multifactorial de los TME. Para el caso específico de las ubicaciones anatómicas de cuello, hombros y manos/muñecas, existe una fuerte relación con la adopción de posturas forzadas o estáticas. ${ }^{31-36}$ También existe una relación causal con altos niveles de repetitividad en el trabajo ${ }^{31,34,35,37,38}$, así como el elevado tiempo dedicado a tareas con una alta repetitividad está claramente asociado con desórdenes músculo-esqueléticos en el cuello y en los hombros, tal cual como reveló un estudio sobre los desórdenes del cuello y de las extremidades superiores de las mujeres en la industria del procesado de pescado. ${ }^{37} \mathrm{La}$ aplicación de fuerza también parece influir significativamente en el desarrollo de dolencias músculoesqueléticas en cuello, hombros y manos/muñecas. ${ }^{21,38}$

Con respecto a la incidencia de los TME en espalda, los factores de riesgo son: el trabajo físico pesado, los levantamientos de carga, los movimientos enérgicos, las posturas forzadas, la exposición a vibraciones en todo el cuerpo y las posturas estáticas. ${ }^{21}$ Respecto a la asociación entre la adopción de posturas estáticas y los TME en la espalda, no parecen existir evidencias suficientes que la confirmen. ${ }^{21,39,40}$ Una exhaustiva revisión de la literatura sobre la asociación entre trabajos en los que los trabajadores permanecen sentados más de la mitad de su jornada y la presencia de dolencias lumbares, llevó a la conclusión que permanecer sentado no constituye por sí solo un factor de riesgo, pero que dicha posición sí supone riesgo en combinación con otros factores, como la adopción de posturas forzadas. ${ }^{40}$ Dicha conclusión coincide con la expuesta por Bruce, que considera que no existen evidencias suficientes que confirmen el riesgo de padecer TME en la espalda ante la adopción únicamente de posturas estáticas.

Los resultados obtenidos en este estudio sugieren focalizar en intervenciones que eviten la aparición de TME ocupacionales y obligan a incluir este tema como prioritario y a profundizar en su origen, factores asociados y prevención. Así pues, es importante realizar una valoración del puesto de trabajo para detallar las situaciones expresadas en el presente estudio para determinar los factores de riesgo no considerados, y lograr así implementar medidas de control que prevengan el desarrollo de los TME en estas trabajadoras. Asimismo, se recomienda analizar condiciones extra-laborales y correlacionar con la morbilidad.

Igualmente sería de valiosa importancia ampliar el estudio al resto de trabajadores de la industria de fabricación de productos plásticos, así como a los trabajadores del mismo sector productivo en otras empresas.

Finalmente, la metodología propuesta en este estudio puede constituir una herramienta de bajo costo, fácil de implementar en los programas de vigilancia epidemiológica músculo-esquelética viable para realizar un diagnóstico en la población y a identificar factores físicos involucrados en el origen de los TME ocupacionales. 
1. Guitart J, Giménez-Crouseilles J. Prevalencia de la tensión muscular elevada, evaluada con un método semiobjetivo y estudio de factores asociados a la misma en una población reumatológica. Rev Soc Esp Dolor. 2002;9:5-12.

2. Wahlström J. Ergonomics, musculoskeletal disorders and computer work. Occup Med. 2005 May;55(3):168-76.

3. Colombia. Ministerio de la Protección Social. Guia de Atención Integral basada en la Evidencia para Desórdenes Musculoesqueléticos (DME) relacionados con Movimientos Repetitivos de Miembros Superiores (Sindrome de Túnel Carpiano, Epicondilitis y Enfermedad de De Quervain) (GATI-DME). Bogotá: Ministerio de la Protección Social; 2006. p. 3-136. (Report, 98067).

4. Colombia. Ministerio de la Protección Social. Guia de Atención Integral basada en la Evidencia para Dolor Lumbar Inesperado y Enfermedad Discal Relacionados con la Manipulación Manual de cargas y otros factores de riesgo en el lugar de Trabajo (GATI-DLI-ED). Bogotá: Ministerio de la Protección Social; 2006. p. 3-134.

5. Colombia. Ministerio de la Protección Social. Guia de Atención Integral basada en la Evidencia para Hombro Doloroso (GATI-HD) relacionado con factores de riesgo en el Trabajo. Bogotá: Ministerio de la Protección Social; 2006. p. 2-124.

6. Falcon D. El $90 \%$ de las enfermedades ocupacionales es por trastornos músculo-esqueléticos [en línea]. Caracas: INPSASEL; 2013 [citado may 2015]. Disponible en: http://www.inpsasel.gob.ve/moo_news/Prensa_722.html

7. Venezuela. Instituto Nacional de Prevención, Salud y Seguridad Laboral (INPSASEL). Estadistica de enfermedades ocupacionales. Caracas: INPSASEL; 2006.

8. Punnett L, Wegman DH. Work-related musculoskeletal disorders: the epidemiologic evidence and the debate. Jelekin [on line] 2004 [cited 2013 Sep 22];14 (1):13-23. Available from: http://www.ncbi.nlm.nih.gov/pubmed/14759746

9. Agencia Europea para la Seguridad y la Salud en el Trabajo. Aligera la carga. Magazine (Luxemburgo). 2007; 10:1-49.

10. Landsbergis $P$. The changing organization of work and the safety and health of working people: a commentary. Occup Env Med [on line]. 2003 [cited 2015 May]; 45(1):61-72. Available from: http://www.ncbi.nlm.nih.gov/pubmed/12553180

11. Guerrero J, York I. Productividad, trabajo y salud: la perspectiva psicosocial. Rev colomb psicol. 2007;16 (01215469): 203-34.

12. Comisión Europea. Libro verde. Bruselas: Comisión Europea; 2013.

13. MAPFRE. Guía básica de seguridad en industrias de plásticos. Madrid: MAPFRE-CAJAMADRID EMPRESAS; 2007.

14. Bryce DM. Plastic injection molding: manufacturing process fundamentals. In: Society of Manufacturing Engineers, editor. Material Selection and Product Design. Vol. II. Maine: Society of Manufacturing Engineers; 1996.

15. Valdés I, Sánchez S. Moldeo por inyección de termoplásticos. México: Limusa; 2003.

16. Fernandes RDCP, Assunção AA, Silvany Neto AM, Carvalho FM. Musculoskeletal disorders among workers in plastic manufacturing plants. Rev Bras Epidemiol [on line]. 2010 [cited 2015 May];13(1):11-20. Available from: http://www.ncbi. nlm.nih.gov/pubmed/20683551

17. Nordander C, Ohlsson K, Akesson I, Arvidsson I, Balogh I, Hansson G-A, et al. Risk of musculoskeletal disorders among females and males in repetitive/ constrained work. Ergonomics [on line]. 2009 [cited 2013 Jun 7];52(10):122639. Available from: http://www.ncbi.nlm.nih.gov/pubmed/19787502

18. Fundación para la Prevención de Riesgos Laborales. Detención de la sobrecarga fisica desde la variable de género; Cuestionarios. Barcelona: Fundación para la Prevención de Riesgos Laborales; 2007. p. 59.

19. Ladou J. Health Effects of Shift Work. West J Med. 1982;6(137):525-30.

20. Mathers C. Global Burden of Disease: data sources, methods and results 2003. Geneva: WHO; 2004.

21. Bruce B. editor. Musculoskeletal Disorders and Workplace Factors. Cincinnati: NIOSH; 1997.

22. Berg $M$, Sandén $A$, Torell $G$, Järvholm B. Persistence of musculoskeletal symptoms: a longitudinal study. Ergonomics [on line]. 1988 [cited 2014 Aug 23];31(9):1281-5. Available from: http://www.ncbi.nlm.nih.gov/pubmed/3191905

23. Palomino J, Ruiz F, Navarro G, Dongo F, Llap P, Gomero R. El trabajo de turnos como factor de riesgo para lumbago en un grupo de trabajadores peruanos. Rev Med Hered. 2005;16:184-9.
24. Dickinson CE, Campion K, Foster AF, Newman SJ, O'Rourke AMT, Thomas PG. Questionnarie development: an examination of the nordic musculoskeletal questionnarie. Appl Ergon. 1992;23(3):197-201.

25. Kuorinka I, Jonsson B, Kilbom a, Vinterberg $H$, Biering-Sørensen $F$, Andersson $\mathrm{G}$, et al. Standardised Nordic questionnaires for the analysis of musculoskeletal symptoms. Appl Ergon [on line]. 1987 [cited 2015 May];18(3):233-7. Available from: http://www.ncbi.nlm.nih.gov/pubmed/15676628

26. Tortosa L, Garcia Molina C, Page A, Ferreras A, Castelló P, Piedrabuena A. Trabajo y envejecimiento. Mejora de las condiciones ergonómicas de la actividad laboral para la promoción de un envejecimiento saludable. Rev Inst Nac Segur Hig Trab. 2004;30:29-36.

27. Landau $K$, Rademacher $H$, Meschke $H$, Winter $G$, Schaub K, Grasmueck $M$, et al. Musculoskeletal disorders in assembly jobs in the automotive industry with special reference to age management aspects. Int J Ind Ergon [on line]. 2008 [cited 2013 Sep 23];28(7-8):561-76. Available from: http://linkinghub.elsevier. com/retrieve/pii/S0169814108000176

28. Vezina N. Rotation implantation: what is at stake? what are the markers? WorkShop Principles and Practices of Job Rotation- CRE-MSD, Centre of Research Expertise for the Prevention of Musculoskeletal Disorders; 2004 Dec 4; Waterloo, Canada. Waterloo: University of Waterloo; 2005. p. 1-22.

29. Sauter SL, Murphy LR, Hurrell JJ, Jr JJH. Factores psicosociales y de organización. Enciclopedia de salud y seguridad en el trabajo. $4^{\text {a }}$. ed. Madrid: Ministerio del Trabajo y Asuntos Sociales; 2001. p. 73.

30. Fernández-Montalvo J, Pinol E. Horario laboral y salud: consecuencias psicológicas de los turnos de trabajo. RPPC. 2000;5(3):207-22.

31. Bonfiglioli R, Mattioli S, Fiorentini C, Graziosi F, Curti S, Violante FS. Relationship between repetitive work and the prevalence of carpal tunnel syndrome in parttime and full-time female supermarket cashiers: a quasi-experimental study. Int Arch Occup Env Hea [on line]. 2007 [cited 2013 Apr 1];80(3):248-53. Available from: http://www.ncbi.nlm.nih.gov/pubmed/ 16865405

32. Skov T, Borg V, Orhede E. Psychosocial and physical risk factors for musculoskeletal disorders of the neck, shoulders, and lower back in salespeople. Occup Env Med [on line]. 1996 [cited 2015 May]; 53(5):35-6. Available from: http:// www.pubmedcentral.nih.gov/articlerender.fcgi?artid $=1128479 \&$ tool=pmcentr ez\&rendertype $=$ abstract

33. Yu IT, Wong TW. Musculoskeletal problems among VDU workers in a Hong Kong bank. Occup Med [on line]. 1996 [cited 2015 May];46(4):275-80. Available from: http://www.ncbi.nlm.nih.gov/pubmed/8854704

34. Vernaza-Pinzón P, Sierra-Torres CH. Dolor Músculo-Esquelético y su Asociación con Factores de Riesgo Ergonómicos en Trabajadores Administrativos. Rev Salud Pública. 2005;7(3):317-26.

35. Natarén J, Noriega E. Los trastornos musculoesqueléticos y la fatiga como indicadores de deficiencias ergonómicas y en la organización del trabajo. Salud Trab. 2004;12(2):27-41.

36. Chandrasakaran a, Chee HL, Rampal KG, Tan GL. The prevalence of musculoskeletal problems and risk factors among women assembly workers in the semiconductor industry. Med J Malaysia [on line]. 2003 [cited 2015 May];58(5):65766. Available from: http://www.ncbi.nlm.nih.gov/pubmed/15190650

37. Ohlsson $\mathrm{K}$, Hansson $\mathrm{G}$ a, Balogh I, Strömberg U, Pålsson B, Nordander C, et al. Disorders of the neck and upper limbs in women in the fish processing industry. Occup Env Med [on line]. 1994 [cited 2015 May];51(12):826-32. Available from: http://www.pubmedcentral.nih.gov/articlerender.fcgi?artid=1128124\&t ool=pmcentrez\&rendertype $=$ abstract

38. Piedrahita $\mathbf{L H}$. Evidencias epidemiológicas entre factores de riesgo en el trabajo y los desórdenes músculo-esqueléticos. Mapfre medicina. 2004;15(3):212-221.

39. Hartvigsen J, Bakketeig LS, Leboeuf-Y de C, Engberg M, Lauritzen T. The association between physical workload and low back pain clouded by the "healthy worker". Ugeskr Laeger. 2002;164:2765-2768.

40. Lis AM, Black KM, Korn H, Nordin M. Association between sitting and occupational LBP. Eur Spine J [on line]. 2007 [cited 2014 Aug 12];16(2):283-98. Available from: http://www.pubmedcentral.nih.gov/articlerender.fcgi?artid=22 00681\&tool=pmcentrez\&rendertype $=$ abstract 